\title{
Discovery potential for directional Dark Matter detection with nuclear emulsions
}

\author{
NEWSdm Collaboration
}

\author{
N. Agafonova ${ }^{1}$, A. Aleksandrov ${ }^{2,3}$, A. Anokhina ${ }^{4}$, T. Asada ${ }^{5}$, V. V. Ashikhmin ${ }^{1}$, I. Bodnarchuk ${ }^{6}$, A. Buonaura ${ }^{2,3}$, \\ M. Chernyavskii ${ }^{7}$, A. Chukanov ${ }^{6}$, N. D’Ambrosio ${ }^{8}$, G. De Lellis ${ }^{2,3}$, A. Di Crescenzo ${ }^{2,3, a}{ }_{\mathbb{D}}$, N. Di Marco ${ }^{8, b}$, \\ S. Dmitrievski ${ }^{6}$, R. I. Enikeev ${ }^{1}$, R. A. Fini ${ }^{9}$, G. Galati ${ }^{2,3}$, V. Gentile ${ }^{10}$, S. Gorbunov ${ }^{7}$, Y. Gornushkin ${ }^{6}$, A. M. Guler ${ }^{11}$, \\ H. Ichiki ${ }^{5}$, T. Katsuragawa ${ }^{5}$, N. Konovalova ${ }^{7}$, K. Kuge ${ }^{12}$, A. Lauria ${ }^{2,3}$, K. Y. Lee ${ }^{13}$, L. Lista ${ }^{2}$, A. S. Malgin ${ }^{1}$, \\ A. Managadze $^{4}$, P. Monacelli ${ }^{14}$, M. C. Montesi ${ }^{2,3}$, T. Naka ${ }^{5}$, N. Okateva ${ }^{7}$, B. D. Park ${ }^{13}$, D. Podgrudkov ${ }^{4}$, \\ N. Polukhina ${ }^{7,15}$, F. Pupilli ${ }^{16,17}$, T. Roganova ${ }^{4}$, A. Rogozhnikov ${ }^{18}$, G. Rosa ${ }^{14,19}$, O. G. Ryazhskaya ${ }^{1}$, O. Sato ${ }^{5}$, \\ I. R. Shakiryanova ${ }^{1}$, T. Shchedrina ${ }^{7}$, C. Sirignano ${ }^{16,17}$, J. Y. Sohn ${ }^{13}$, A. Sotnikov ${ }^{6}$, N. Starkov ${ }^{7}$, P. Strolin St, $^{2,}$ \\ V. Tioukov ${ }^{2,3}$, A. Umemoto ${ }^{5}$, A. Ustyuzhanin ${ }^{18,20}$, C. S. Yoon ${ }^{13}$, M. Yoshimoto ${ }^{5}$, S. Vasina ${ }^{6}$ \\ ${ }^{1}$ INR RAS-Institute for Nuclear Research of the Russian Academy of Sciences, Moscow, Russia \\ 2 INFN Sezione di Napoli, Naples, Italy \\ ${ }^{3}$ Dipartimento di Fisica dell’Università Federico II di Napoli, Naples, Italy \\ ${ }^{4}$ INP MSU-Skobeltsyn Institute of Nuclear Physics of Moscow State University, Moscow, Russia \\ ${ }^{5}$ Nagoya University and KM Institute, Nagoya, Japan \\ ${ }^{6}$ JINR-Joint Institute for Nuclear Research, Dubna, Russia \\ ${ }^{7}$ LPI-Lebedev Physical Institute of the Russian Academy of Sciences, Moscow, Russia \\ 8 INFN-Laboratori Nazionali del Gran Sasso, Assergi, L'Aquila, Italy \\ ${ }^{9}$ INFN Sezione di Bari, Bari, Italy \\ ${ }^{10}$ Gran Sasso Science Institute, L'Aquila, Italy \\ ${ }^{11}$ METU-Middle East Technical University, Ankara, Turkey \\ 12 Chiba University, Chiba, Japan \\ 13 RINS and Department of Physics Education, Gyeongsang National University, Jinju, Korea \\ 14 INFN Sezione di Roma, Rome, Italy \\ 15 National Research Nuclear University, Moscow Engineering Physical University, Moscow, Russia \\ 16 INFN Sezione di Padova, Padua, Italy \\ 17 Dipartimento di Fisica e Astronomia dell'Università di Padova, Padua, Italy \\ 18 Yandex School of Data Analysis, Moscow, Russia \\ ${ }^{19}$ Dipartimento di Fisica dell’Università di Roma, Rome, Italy \\ ${ }^{20}$ National Research University, Higher School of Economics, Moscow, Russia
}

Received: 22 January 2018 / Accepted: 11 July 2018 / Published online: 17 July 2018

(C) The Author(s) 2018

\begin{abstract}
Direct Dark Matter searches are nowadays one of the most fervid research topics with many experimental efforts devoted to the search for nuclear recoils induced by the scattering of Weakly Interactive Massive Particles (WIMPs). Detectors able to reconstruct the direction of the nucleus recoiling against the scattering WIMP are opening a new frontier to possibly extend Dark Matter searches beyond the neutrino background. Exploiting directionality would also prove the galactic origin of Dark Matter with an unambiguous signal-to-background separation. Indeed, the angular distribution of recoiled nuclei is centered around the direction of
\end{abstract}

\footnotetext{
a e-mail: antonia.dicrescenzo@na.infn.it

b e-mail: natalia.dimarco@lngs.infn.it
}

the Cygnus constellation, while the background distribution is expected to be isotropic. Current directional experiments are based on gas TPC whose sensitivity is limited by the small achievable detector mass. In this paper we present the discovery potential of a directional experiment based on the use of a solid target made of newly developed nuclear emulsions and of optical read-out systems reaching unprecedented nanometric resolution.

\section{Introduction}

Although its nature remains undisclosed, there are indications that the Dark Matter (DM) is made by hypothetical 
particles called WIMPs (Weakly Interacting Massive Particles). Current experimental efforts in the field of direct DM searches are devoted to the detection of the rare interactions of WIMPs from the galactic halo with nuclei in a terrestrial detector.

As DM detectors are rapidly improving in sensitivity, at some point they will encounter the so-called "neutrino floor" where coherent scattering of Solar, atmospheric, and diffuse supernovae neutrinos creates an irreducible background. Neutrinos are therefore the ultimate background for WIMP direct detection searches as they produce recoils with similar rates and energy spectra.

New generation detectors capable of measuring the direction of nuclear recoils induced by WIMP elastic scatterings would provide an unambiguous identification of WIMPs as candidate for the galactic DM and could overcome the limit imposed by coherent neutrino scattering.

Several directional approaches have been proposed [1]. Experiments based on the use of low pressure gaseous Time Projection Chambers (TPCs) typically make use of compounds rich in ${ }^{19} \mathrm{~F}$ nucleus in order to set limits on the SpinDependent (SD) coupling of WIMPs with less than $\mathrm{kg}$ mass detectors [2]. Nevertheless this technology is hardly scalable to very large detector masses needed to reach a good sensitivity to the Spin-Independent (SI) case.

The use of a solid target for directional searches would overcome the mass limitation of the gaseous TPC approach thus allowing to reach a high sensitivity in the low cross section sectors of the SI case. Nevertheless, in a solid medium, the track of the WIMP-scattered nuclear recoil will have a path length of the order of a few 100 nanometers. A detector with unprecedented high tracking resolution is therefore needed: the approach proposed by the NEWSdm Collaboration consists of using a nuclear emulsion-based detector acting both as target and as tracking device. The project foresees the use of a novel emulsion technology called Nano Imaging Tracker (NIT) $[3,4]$ featuring a position resolution an order of magnitude higher than that of the emulsion used in the OPERA experiment $(\sim 1 \mu \mathrm{m})$ [5]. The detector is made of a stack of NIT emulsion films surrounded by a shield to reduce the external background. The detector is then placed on an equatorial telescope in order to keep fixed the detector orientation with respect to the incoming apparent WIMP flux, i.e. toward the Cygnus constellation, thus compensating the Earth rotation. The emulsion films are placed in such a way that their surface is permanently parallel to the Galactic plane. The angular distribution of the WIMP-scattered nuclei is therefore expected to be strongly anisotropic with a peak centered in the opposite direction to the motion of the Solar System in the Galaxy. A detailed description of the NEWSdm experiment can be found in Ref. [6]. The capability to detect nuclear recoils like those induced by a WIMP scattering was demonstrated with NIT emulsion films and an intrinsic angular resolution of $13^{\circ}$ was measured [6].

In this paper we describe the potentialities of an emulsionbased detector as foreseen by the NEWSdm experiment, that is capable of distinguishing the anisotropic WIMP signal over the isotropic background. We report the discovery potential and the exclusion limit for a typical detector mass per exposure time of $100 \mathrm{~kg} \cdot$ year and we give future prospects towards the overcome of the neutrino background limit.

\section{Signal and background modelling}

\subsection{Dark Matter model}

We base our analysis on the hypothesis of a Standard Halo Model (SHM) describing the form of the DM distribution. For a spherically symmetric-isothermal DM halo, with density profile $\rho(r) \propto r^{-2}$, the resulting velocity distribution in the reference frame of the Galaxy has a MaxwellianBoltzmann form [7]

$f(\mathbf{v})=\frac{1}{\left(2 \pi \sigma_{v}^{2}\right)^{1 / 2}} \exp \left(-\frac{\mathbf{v}^{2}}{2 \sigma_{v}^{2}}\right)$,

with velocity dispersion $\sigma_{v}=v_{0} / \sqrt{2}$ and $v_{0} \simeq 220 \mathrm{~km} \cdot \mathrm{s}^{-1}$. A cut-off in the distribution at the Galactic escape velocity $v_{\text {esc }} \simeq 544 \mathrm{kms}^{-1}$ is considered.

We take a detector velocity equal to the tangential component of the Sun motion around the Galactic center $v_{\text {Earth }}=$ $220 \mathrm{~km} \cdot \mathrm{s}^{-1}$, and we neglect the Sun peculiar velocity with respect to the Local Standard of Rest (LSR) and the Earth orbital velocity around the Sun, their contribution being smaller than the uncertainty on the Sun velocity [8].

\subsection{Signal and background detection}

In this work we consider the realistic NEWSdm detector with a $100 \mathrm{~nm}$ threshold and a 2D track reconstruction capability, as proven by test beam results reported in [6]. Studies are ongoing to further reduce the threshold, as outline in the last section of this work. Additional improvements devoted to exploit the intrinsic emulsion capability of recording 3D tracks with nanometric accuracy are the subject of a future experimental program [9] and are not taken into account in the present work.

The track reconstruction is performed in the $x y$ Galactic plane, where the $x$-axis is directed opposite to the Cygnus constellation (see Fig. 1). In this reference system, the angle of the nuclear recoil $(\theta)$ is defined as the angular difference between its projection in $x y$-plane and the $x$-axis. Assuming no head-tail sense recognition, the $2 \mathrm{D}$ angle is lies in the $[-\pi / 2, \pi / 2]$ range. 


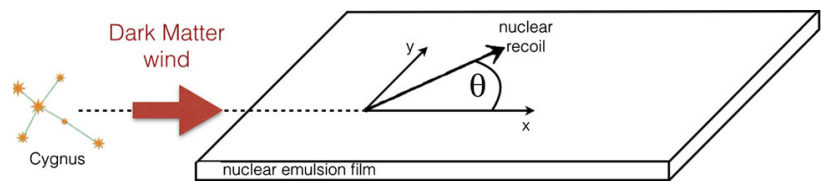

Fig. 1 The 2D reconstruction is performed in the $x y$-plane. The $x$ axis is directed opposite to the Cygnus constellation and $\theta$ is the angle between the $x$-axis and the projection of the nuclear recoil in the $x y$ plane

The angular distribution of the recoiled nuclei is expected to have a Gaussian shape peaked at zero, with standard deviation $\left(\sigma_{\theta}^{W n-\text { scatt }}\right)$ that decreases as the WIMP mass increases. The lighter the WIMP, the stronger the angular anisotropy. Indeed, due to the fact that low WIMP mass induce an energy distribution shifted to low energy, events above 100 $\mathrm{nm}$ threshold are those with the most pronounced directional feature.

As far as the background is concerned, the contribution from $\beta$-rays is considered negligible. Indeed, at temperatures around $100 \mathrm{~K}$, NIT emulsion films become insensitive to electrons while keeping sufficiently good sensitivity to ions. The reason for such a result is the suppression at low temperature of one mechanism to produce latent image specs, i.e. the formation of interstitial silver ions producing silver grains after the chemical development. This is the only mechanism occurring for electrons. On the other side, given the higher energy deposition of nuclear recoils, atomic displacements are induced and phonons are transmitted to the crystals, heating them up and producing latent image specs. This result is illustrated in the Ref. [10]. The electron background is therefore neglected.

The background from neutrons is usually considered as irreducible, since they induce nuclear recoils as WIMPs do. Neutrons in underground laboratories originate from cosmic muon interactions, environmental radioactivity, spontaneous fissions and $(\alpha, \mathrm{n})$ reactions. The first two sources can be reduced by an appropriate shielding, the latter one comes from the intrinsic radioactivity of the target materials. The measurements performed by the Collaboration on NIT samples with ICP-MS and gamma spectroscopy techniques together with a dedicated simulation show that the detectable neutron induced background would be of the order of 0.06 $\mathrm{n} / \mathrm{kg} /$ year [11]. The most radioactive compound in the emulsion is the gelatine which, given the mass fraction, provides about $1 / 3$ of the total neutron yield. The Collaboration plans to replace the gelatine with synthetic polymers. This will make the background from this emulsion compound negligible, thus reducing the radioactivity to $2 / 3$ of the current value. We assume that a careful selection of the raw materials and the corresponding production processes could possibly reduce $\mathrm{AgBr}$ radioactivity down to about $10 \%$ of the current value. This would result in $0.004 \mathrm{n} / \mathrm{kg} /$ year that will be sufficient to operate a $100 \mathrm{~kg}$ year scale detector with a negligible background. It is worth noting that the contribution from intrinsic radioactivity is associated with an isotropic distribution in the laboratory frame.

The directional detection has the unique capability of distinguishing the WIMP signal from the background by exploiting the feature of the signal, expected to be peaked in the opposite direction to the motion of the Sun.

\subsection{Recoil simulation}

Nuclear recoil tracks produced in WIMP interactions show deviations from their original path due to continuous collisions with atoms in the target. In order to quantify these deviations we evaluated the effect of the straggling in an emulsion target made of the following nuclei (mass fraction in \%): $\mathrm{H}$ (1.6), C (10.1), N (2.7), O (7.4), S (0.3), Br (32.0), Ag (44.0), I (1.9). The total density is $3.43 \mathrm{~g} / \mathrm{cm}^{3}$. When a charged particle passes through the emulsion film, a number of $\mathrm{AgBr}$ grains per unit path length are sensitised. Measurements performed with both a SEM and an X-ray microscope showed that heavy nuclei produce on average 12 grains $/ \mu \mathrm{m}$ in NIT emulsions.

The study was performed with the SRIM [12] software package and its TRIM (Transport of Ions in Matter) track generator Monte Carlo program.

All nuclear recoils were simulated in the energy range from 0 to $500 \mathrm{keV}$. All the tracks with at least $100 \mathrm{~nm}$ length were considered. The range was calculated using the coordinates of the first and last point of the ion track. The energy threshold corresponding to the minimum track length was evaluated from the average range estimated by SRIM. It ranges from $25 \mathrm{keV}$ for Carbon to $273 \mathrm{KeV}$ for Iodine. Being NIT emulsions less sensitive to protons, $\mathrm{H}$ recoils are conservatively not taken into account.

In the above mentioned DM model, the mean direction of the track was compared with the initial direction of the recoil: the difference of the two directions shows a Gaussian distribution centered at zero, with a sigma $\left(\sigma_{\theta}^{\text {straggl }}\right)$ that depends on the selected nuclei and on the recoil energy and reflects the loss of the directional information due to the straggling in the target. Figure 2 shows $\sigma_{\theta}^{\text {straggl }}$ as a function of the recoil energy for the most abundant nuclei in emulsion. Only ranges above $100 \mathrm{~nm}$ are considered. The angular deviation due to straggling ranges from 0.3 to $0.5 \mathrm{rad}$ for light nuclei $(\mathrm{C}, \mathrm{N}, \mathrm{O})$ and from 0.2 to 0.35 for heavy nuclei (Ag, Br).

Figure 3 shows the overall angular deviation expected for nuclear recoils in an emulsion target as a function of the WIMP mass. Black dots represent the angular deviation of nuclear recoils from the direction of Earth's motion, as it arises from the WIMP-nucleus scattering $\left(\sigma_{\theta}^{W n-s c a t t}\right)$, the blue triangles represent the angular deviation due to straggling in emulsion target only $\left(\sigma_{\theta}^{\text {straggl }}\right)$, the red boxes are the 


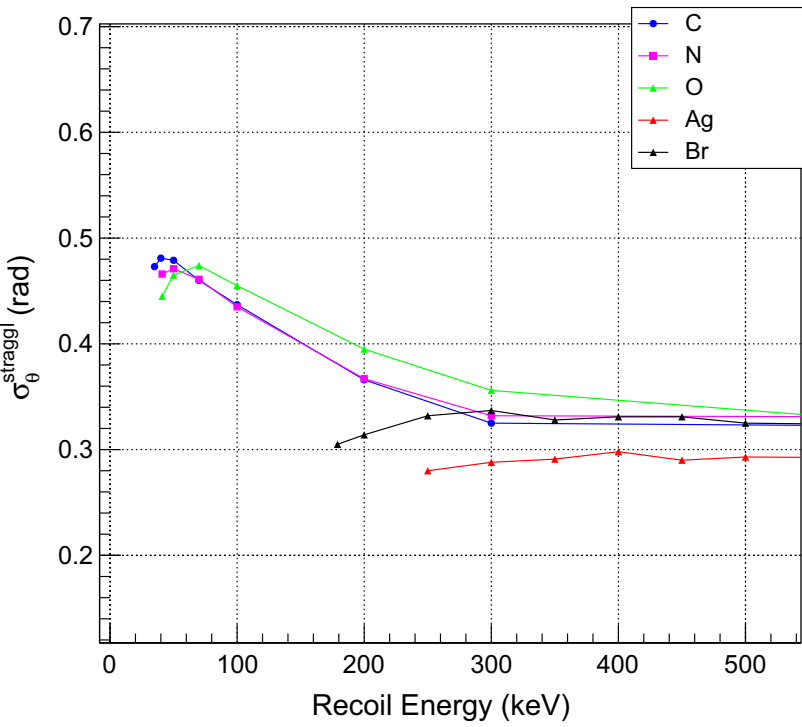

Fig. 2 Angular deviation due to the straggling of nuclei in an emulsion target. A $100 \mathrm{~nm}$ threshold on the range of the recoil track is applied

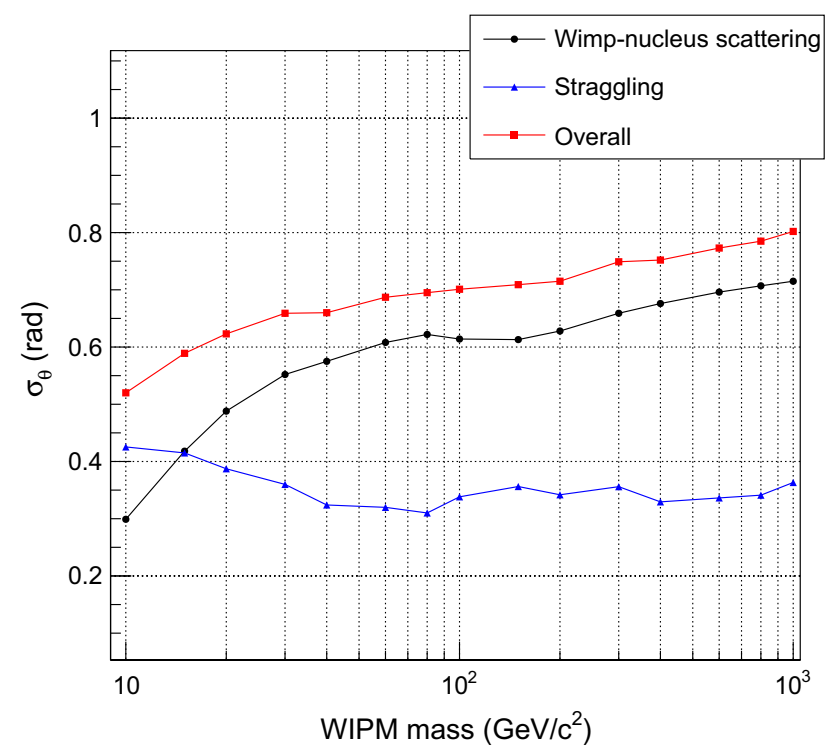

Fig. 3 Overall angular deviation of nuclear recoils in an emulsion target as a function of the WIMP mass (red boxes). The two components due to the WIM-nucleus scattering (black dots) and the straggling (blue triangles) are also shown

convolution of the two components, hereafter referred to as $\sigma_{\theta}^{T O T}$

$$
\sigma_{\theta}^{T O T}=\sqrt{\left(\sigma_{\theta}^{\text {Wn-scatt }}\right)^{2}+\left(\sigma_{\theta}^{\text {straggl }}\right)^{2}} .
$$

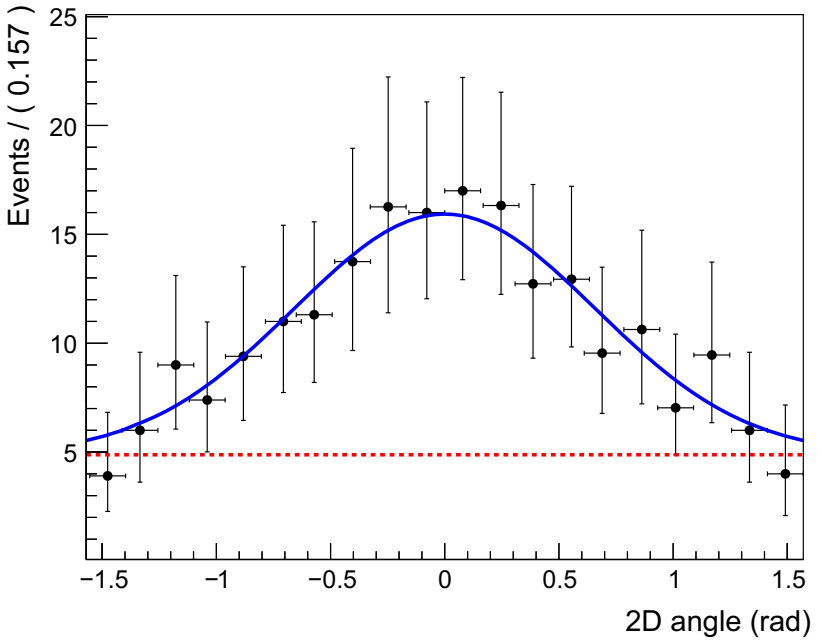

Fig. 4 2D angular distribution of 100 WIMP-induced recoils and 100 background events. Signal and background components are represented as solid blue and dashed red lines, respectively. Recoils are produced for an emulsion target, a $40 \mathrm{GeV} / \mathrm{c}^{2}$ mass WIMP and a $100 \mathrm{~nm}$ threshold

\section{Signal extraction}

We used the variable $\sigma_{\theta}^{T O T}$ as a function of the incident WIMP mass to evaluate the capability of an emulsion directional detector to distinguish the WIMP-induced signal from the expected isotropically distributed background.

The estimate of the expected significance of a directional emulsion experiment was performed in a frequentist approach with the Profile Likelihood ratio test, taking into account the characterisation of the signal angular spectrum, as a function of the incident WIMP mass. In the Profile Likelihood ratio test [13] the null hypothesis $H_{0}$ (background only) is tested against the alternative hypothesis $H_{1}$ including both signal and background. We have considered the extended likelihood function

$$
\begin{aligned}
\mathscr{L}= & \frac{\left(\mu_{s}+\mu_{b}\right)^{N}}{N !} e^{-\left(\mu_{s}+\mu_{b}\right)} \\
& \times \prod_{i=1}^{N}\left(\frac{\mu_{s}}{\mu_{s}+\mu_{b}} S\left(R_{i}\right)+\frac{\mu_{b}}{\mu_{s}+\mu_{b}} B\left(R_{i}\right)\right),
\end{aligned}
$$

where $\mu_{b}$ and $\mu_{s}$ are the number of expected background and WIMP events, respectively; $N$ is the total number of observed events, $R_{i}$ is the direction of each event while the functions $S$ and $B$ are the probability density functions (PDF) for signal and background, respectively. Figure 4 shows an example of the model used to describe signal and background: the signal PDF is a Gaussian distribution of the 2D angular recoils for a WIMP mass of $40 \mathrm{GeV} / \mathrm{c}^{2}$ (blue curve) while the background PDF is a uniform distribution (dashed red line). Both distributions are normalized to 100 events. 
The statistical model was implemented within the RooStats tool [14], whose classes are built on top of the RooFit [15] package of the ROOT framework [16].

\section{Discovery potential}

In the following we consider a $100 \mathrm{~kg} \cdot$ year NEWSdm detector with a recoil track length range above $100 \mathrm{~nm}$ and no head-tail sense recognition.

We studied the effect of background/signal contribution to data. Figure 5 shows the behavior of the mean significance as a function of the data purity $\lambda=\mu_{s} /\left(\mu_{s}+\mu_{b}\right)$, i.e. the expected fraction of signal events in the data, for three different numbers of observed events $N=10,50,100$, for a WIMP mass of $40 \mathrm{GeV} / \mathrm{c}^{2}$. As expected the mean significance is an increasing function of $\lambda$. In addition, for a fixed $\lambda$, increasing the total number of events determines an improvement in the significance. For a given value of $\lambda=0.5$, for example, the significance of a DM detection can be enhanced from $2 \sigma$ to $6 \sigma$ with an exposure ten times larger. Figure 6 reports the minimum number of signal events required to achieve a $3 \sigma$ evidence as a function of the WIMP mass. Three cases are presented: 1, 10, 100 background events. For comparison, the curves obtained with a counting method based on the Poisson statistics are also reported. The counting method is the most conservative approach for the significance evaluation because it does not assume any knowledge on the shape of both background and signal. In case of a large background contamination $\left(\mu_{b}=100\right)$, at high WIMP masses $\left(\sim 1000 \mathrm{GeV} / \mathrm{c}^{2}\right)$ about 29 signal events are required to claim a $3 \sigma$ evidence while about 25 signal events are enough at low WIMP masses $\left(\sim 6 \mathrm{GeV} / \mathrm{c}^{2}\right)$.

The required number of signal events decreases as the background contamination is reduced, ranging from 11 to 9 events for $\mu_{b}=10$ and from 5 to 4 events for $\mu_{b}=1$.

The $3 \sigma$ curve in the (mass, cross section) plane was obtained for the three background hypotheses, assuming a $100 \mathrm{~kg}$.year exposure: the results obtained with the likelihood ratio test are reported in Fig. 7, together with the ones obtained with the counting method. One can see that a directional detector may lead to an improvement with respect to the counting method ranging from 10 to $20 \%$ ( $8 \%$ to $25 \%$ ) for $\mu_{b}=100\left(\mu_{b}=10\right)$.

\section{Upper limit evaluation}

In case no evidence for DM can be drawn from data, an exclusion limit can be derived. This will be the case for the very first results of an emulsion directional detector with short exposure.

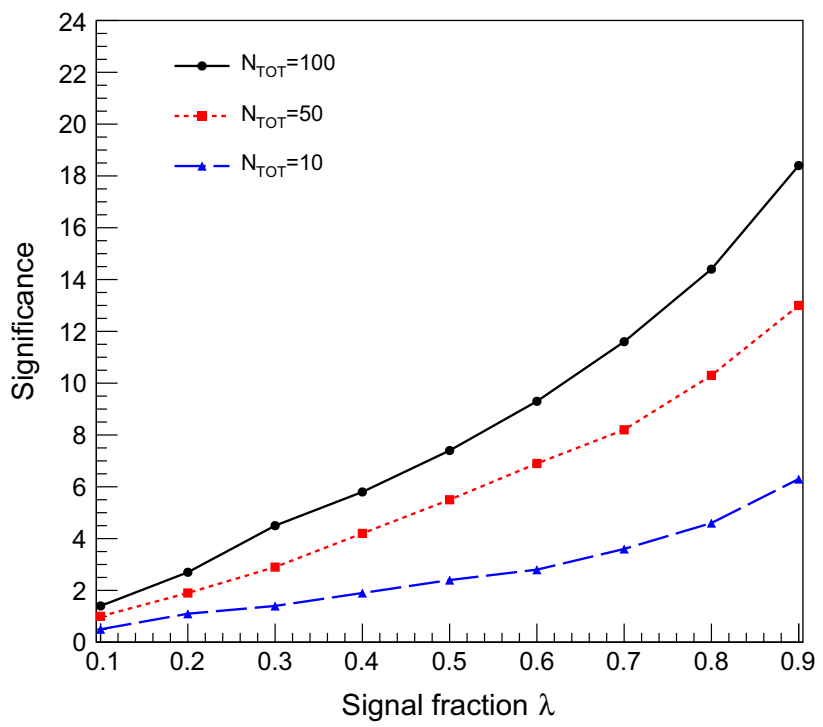

Fig. 5 Mean significance as a function of the expected WIMP fraction $\lambda$ for $N=10$ (black solid line), 50 (red dotted line), 100 (blue dashed line). A $40 \mathrm{GeV} / \mathrm{c}^{2}$ WIMP mass is assumed

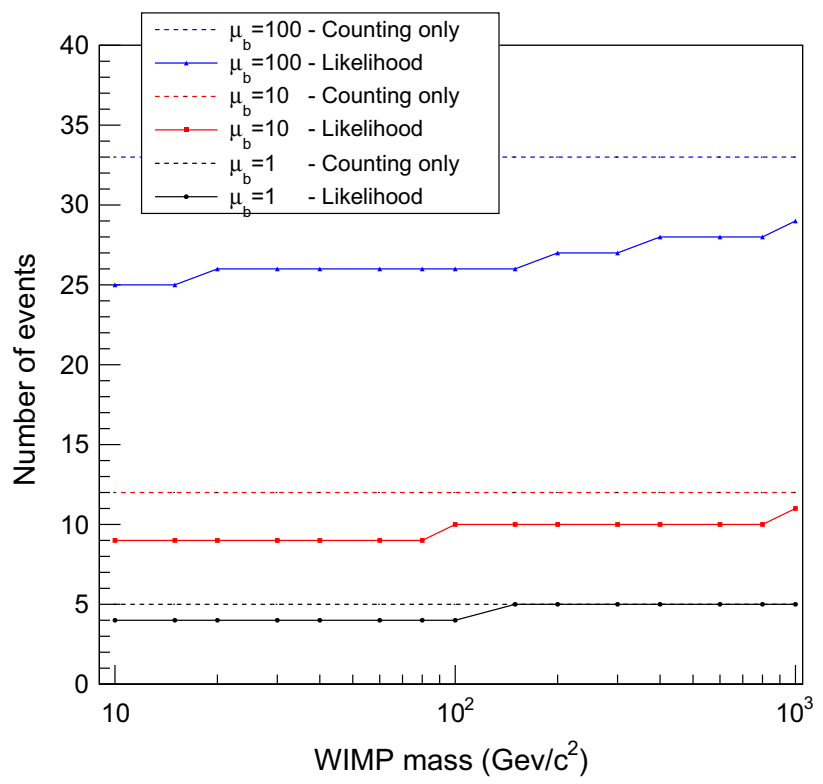

Fig. 6 Minimum number of signal events required to get a $3 \sigma$ evidence as a function of the WIMP mass. Three background hypotheses are considered: 100 (black line), 10 (red line) and 1 (blue line) events. Dotted lines show the results obtained with the counting method

For the upper limit evaluation we have used the Profiled Likelihood Ratio method, with modified frequentist approach (CLs method [17]), and we have fixed the Confidence Level at $90 \%$. The sensitivity curve is achieved by finding the exclusion limits for the different WIMP masses. Figure 8 shows the results of the scan of WIMP masses in the range $[10,1000] \mathrm{GeV} / \mathrm{c}^{2}$ performed with the Likelihood Ratio method. It represents the upper limit to the number of signal events for 100 expected background events, ranging 


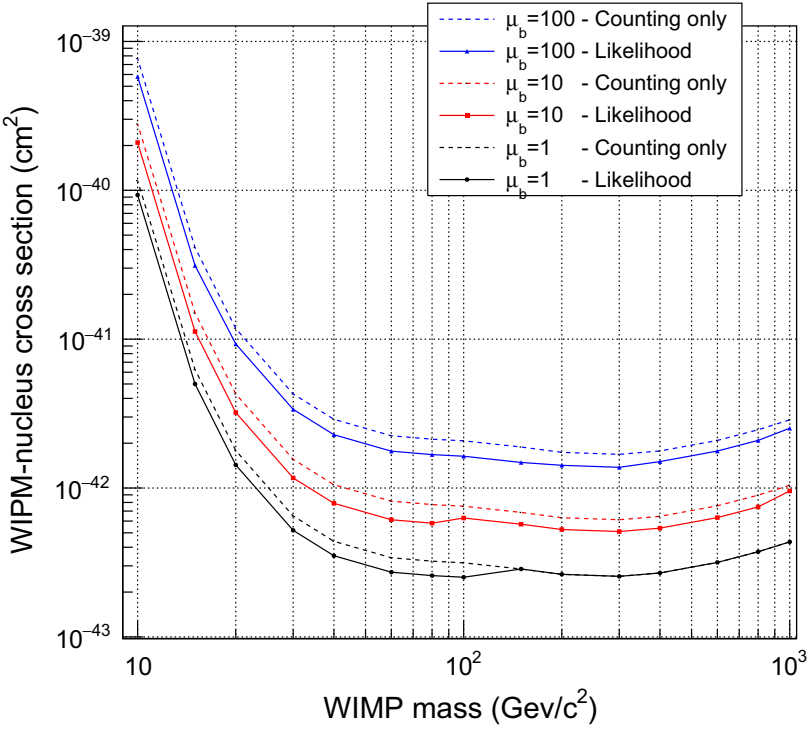

Fig. $73 \sigma$ evidence curve in the (mass, cross section) plane for a $100 \mathrm{~kg} \cdot$ year emulsion detector. Three background hypotheses are considered: 100 (black line), 10 (red line) and 1 (blue line) events. Dotted lines show the results obtained with the counting method

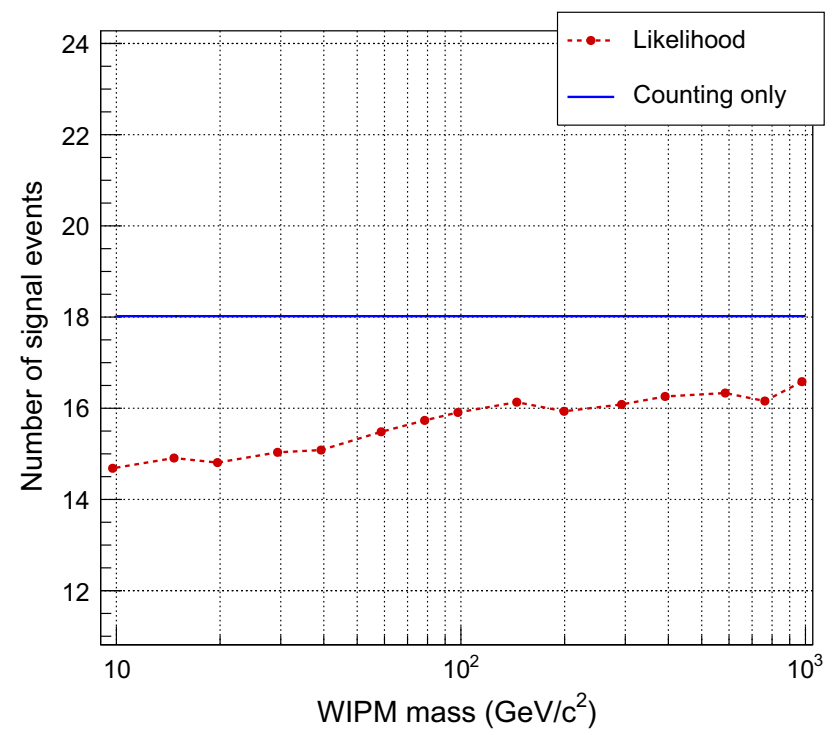

Fig. 8 Upper limit on the number of signal events at 90\% CL for WIMP masses in the $[10,1000] \mathrm{GeV} / \mathrm{c}^{2}$ range for $\mu_{b}=100$. The solid blue line represents the upper limit obtained with the counting method

from about 15-17 events. For comparison, the curve obtained with the counting method is also reported.

The upper limit on the number of signal events can be translated into the exclusion limit in the (mass, cross section) plane. Figure 9 shows the exclusion curve obtained assuming a $100 \mathrm{~kg}$.year exposure with 100 background events. The improvement with respect to the counting method ranges from 10 to $20 \%$.

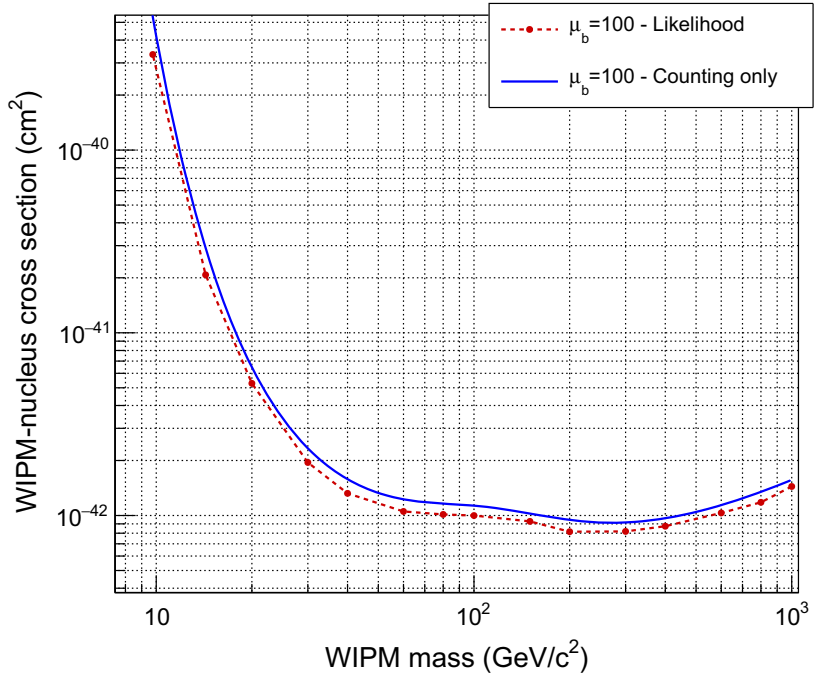

Fig. 9 Exclusion curve at $90 \% \mathrm{CL}$ for WIMP in the (mass, crosssection) plane for a $100 \mathrm{~kg} \cdot y e a r$ emulsion detector and $\mu_{b}=100$. The continuous blue line represents the exclusion curve obtained with the counting method

\section{Dark Matter identification}

Beyond the detection of an excess above the background expectation, the demonstration that the signal is due to Dark Matter stands as a major challenge.

A directional experiment has the unique opportunity to test the anisotropy of the observed signal, thus providing an unambiguous proof of the WIMP origin of the recoil signal. The measurement of the recoil direction is, indeed, a "smoking gun" for the rejection of the isotropic background hypothesis.

We have used a likelihood ratio test to quantitatively estimate the separation between the signal and background hypotheses. The likelihood functions in the background-only hypothesis $H_{0}$ and in the signal hypothesis $H_{1}$ are defined, respectively, as

$$
\begin{aligned}
& \mathscr{L}_{b} \equiv \mathscr{L}\left(x_{1}, \ldots, x_{n} \mid H_{0}\right)=\prod_{i=1}^{N} f\left(x_{i} \mid H_{0}\right) \\
& \mathscr{L}_{s} \equiv \mathscr{L}\left(x_{1}, \ldots, x_{n} \mid H_{1}\right)=\prod_{i=1}^{N} f\left(x_{i} \mid H_{1}\right),
\end{aligned}
$$

where $N$ is the number of observed events, $x_{i}$ the measured recoil direction for the $i$-th event, $f\left(x_{i} \mid H_{0}\right)$ and $f\left(x_{i} \mid H_{1}\right)$ the probability to observe the value $x_{i}$ under the background only and the WIMP only hypothesis, respectively.

We have used the signal characterisation as a function of the WIMP mass reported in Sect. 3, including the straggling effect of nuclear recoils in the emulsion target. 


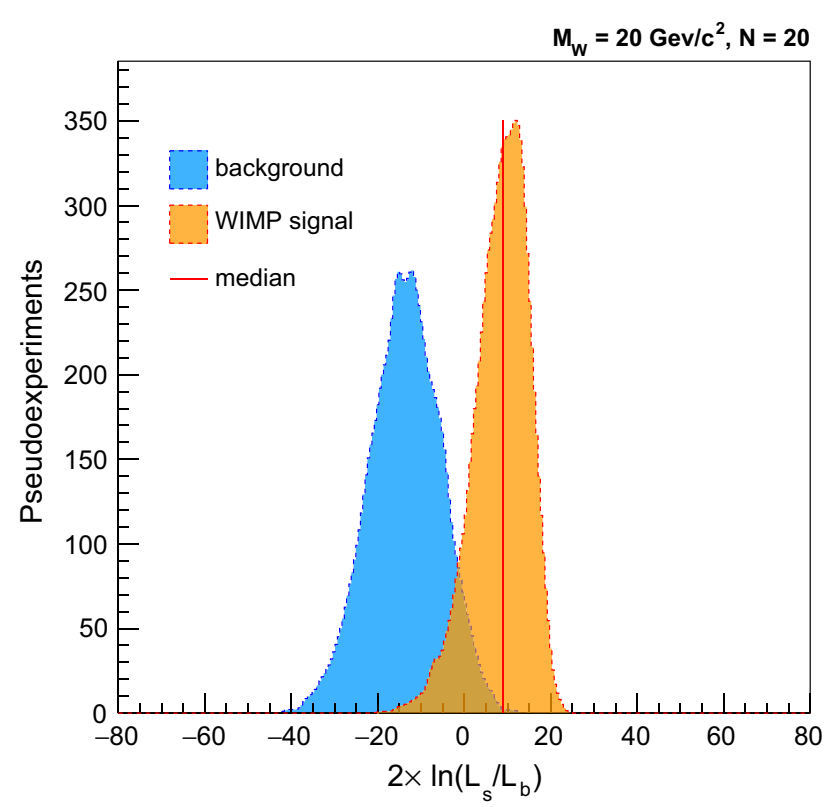

Fig. 10 Distribution of the test statistics $q=2 \ln \left(\mathscr{L}_{s} / \mathscr{L}_{b}\right)$ for the $\mathrm{M}_{W}=20 \mathrm{GeV} / \mathrm{c}^{2}$ WIMP hypothesis tested against the background only hypothesis, assuming 20 observed events. The expectation for the background is represented by the blue histogram on the left and for the signal hypothesis by the orange histogram on the right. The red line indicates the median $q$ value for the WIMP signal

For a given number of observed events $N$ we simulated $10^{4}$ pseudo-experiments and evaluated the test statistics defined as

$q=2 \ln \left(\mathscr{L}_{s} / \mathscr{L}_{b}\right)$

Figure 10 shows the distribution of the test statistics for the signal and background hypotheses, assuming 20 observed events and a WIMP mass of $20 \mathrm{GeV} / \mathrm{c}^{2}$.

The expected separation between the two hypotheses ( $p$ value) is estimated as the fraction of cases in the background hypothesis $\left(H_{0}\right)$ where the test statistic $q$ is above the median value for the WIMP signal $\left(H_{1}\right)$. The $p$-value amounts to $1.27 \times 10^{-3}$, corresponding to a $3 \sigma \mathrm{CL}$ in the rejection of the null hypothesis.

The expected separation for different values of WIMP masses, ranging from 10 to $1000 \mathrm{GeV} / \mathrm{c}^{2}$, is summarized in Fig. 11 assuming 20 (top panel) and 130 (bottom panel) observed events. The median expectation for the Dark Matter signal is represented by the black dots with the green $(68 \% \mathrm{CL})$ and yellow $(95 \% \mathrm{CL})$ solid color regions and for the background hypotheses by the red triangles with the red $(68 \% \mathrm{CL})$ and black $(95 \% \mathrm{CL})$ hatched regions. The observation of 20 (130) events allows to prove that the data are not compatible with the expected background with a $3 \sigma$ CL for WIMP masses below 20 (1000) $\mathrm{GeV} / \mathrm{c}^{2}$.

This highlights the fact that a directional detection approach could lead to the discovery of DM and to the con-
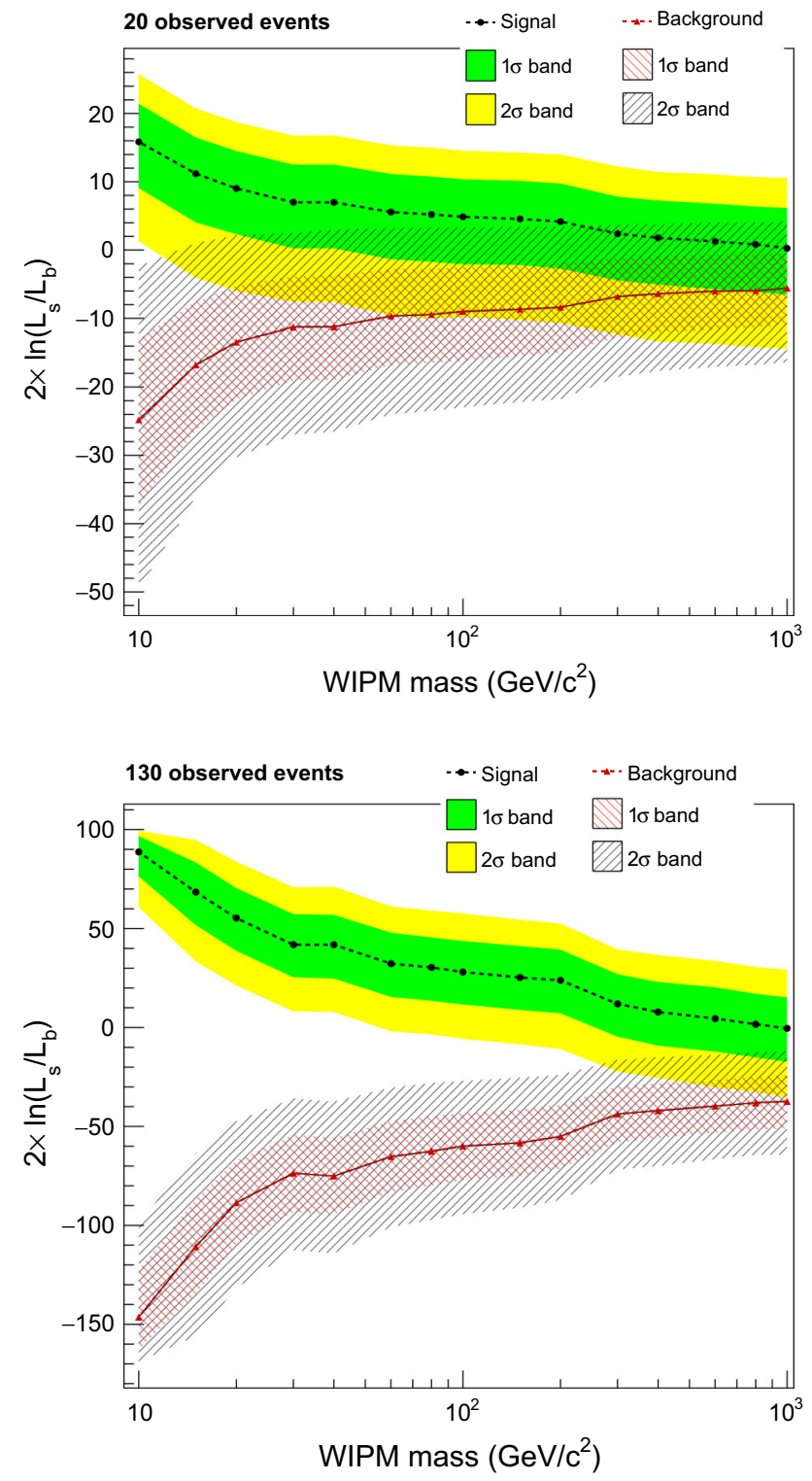

Fig. 11 Test statistic $q$ as a function of the WIMP mass for the signal hypothesis tested against the background hypothesis, assuming 20 (top) or 130 (bottom) observed events

firmation of its galactic origin with a relatively small number of observed event.

\section{NEWSdm towards "neutrino floor"}

In the previous sections it has been shown how the directional information can be used to discriminate DM signal from isotropic background for a realistic NEWSdm detector with $100 \mathrm{~kg} \times$ year exposure and $100 \mathrm{~nm}$ threshold.

The discrimination based on the measurement of the recoil direction offers the unique possibility to search for a DM signal beyond the "neutrino floor", where neutrinos interacting 


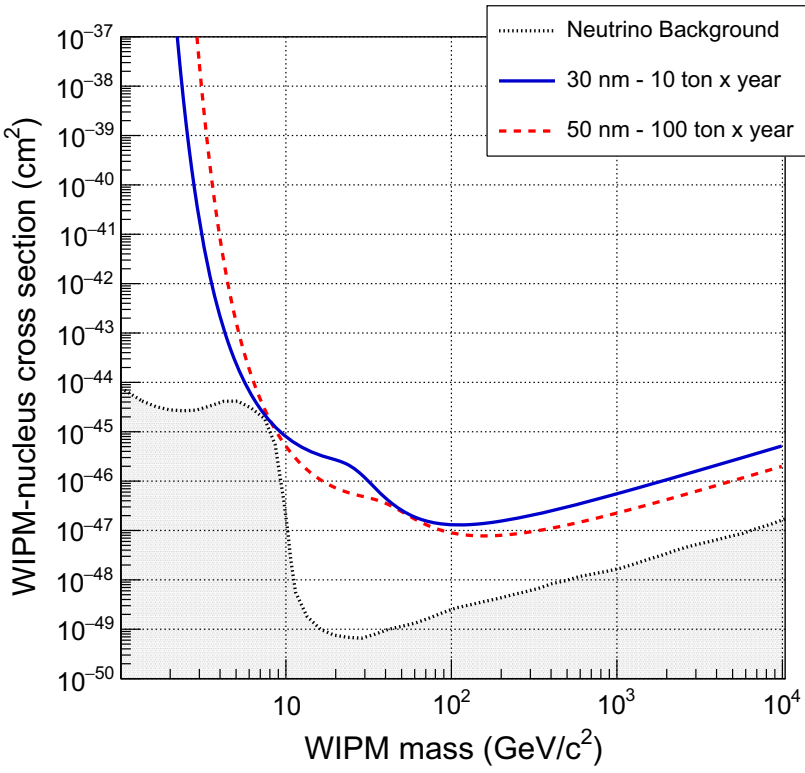

Fig. 12 Exclusion limits for the NEWSdm detector with 10 ton $\times$ year exposure and $30 \mathrm{~nm}$ threshold (solid blue curve) and with 100 ton $\times$ year exposure and $50 \mathrm{~nm}$ threshold (dashed red curve). Zero background is assumed. The gray dotted curve represents the neutrino bound, as evaluated in [18]

coherently with atomic nuclei would produce recoils that cannot be distinguished from DM interactions.

The exploration of the neutrino background region requires both the construction of a larger mass detector and the reduction of the track length threshold. In Fig. 12 the NEWSdm exclusion limit in the zero background hypothesis is compared with the curve representing the neutrino bound for a Xe/Ge target, as evaluated in [18]. The neutrino limit is reached with a $10(100)$ ton $\times$ year exposure if a $30(50) \mathrm{nm}$ threshold is assumed.

Thanks to a new technology based on the use of polarized light with optical microscopes, a $10 \mathrm{~nm}$ accuracy has been already achieved on both $X$ and $Y$ coordinates while keeping the high scanning speed performances of fully automated optical microscopes [6]. Therefore, it is certainly possible to lower the minimum detectable track length beyond $100 \mathrm{~nm}$, provided that the grain size is small enough to build a track within that range. Recently Ultra-Nano Imaging Tracker (UNIT) with a grain size of about $20 \mathrm{~nm}$ have been developed, paving the way for a reduction of the detector threshold.

\section{Conclusions}

The use of fine-grained nuclear emulsions both as target and nanometric tracking device for directional DM searches offers a unique opportunity for a high-significance discovery of galactic DM. Indeed, directional detection is the only way to extend the DM direct detection beyond the "neutrino floor".

In this paper we have evaluated the discovery potential of the NEWSdm detector in the search for WIMPs originated in the galactic halo, assuming a $100 \mathrm{~nm}$ threshold in the track length and the 2D angle reconstruction without headtail sense recognition. A detailed simulation was performed to take into account the straggling effect of nuclear recoils in the target material.

As a result of the continuous improvements in the field of nuclear emulsion technologies, relevant progresses in the reduction of the track length threshold are foreseen in the near future, thus allowing to significantly enhance the explored region in the DM parameter space towards and possibly beyond the "neutrino floor".

Open Access This article is distributed under the terms of the Creative Commons Attribution 4.0 International License (http://creativecomm ons.org/licenses/by/4.0/), which permits unrestricted use, distribution, and reproduction in any medium, provided you give appropriate credit to the original author(s) and the source, provide a link to the Creative Commons license, and indicate if changes were made. Funded by $\mathrm{SCOAP}^{3}$.

\section{References}

1. J.B.R. Battat et al., Phys. Rept. C662, 1 (2016)

2. D.R. Tovey et al., Phys. Lett. B 488, 17 (2000)

3. M. Natsume et al., Nucl. Instr. Meth. A575, 439 (2007)

4. T. Naka et al., Nucl. Instr. Meth. A718, 519 (2013)

5. R. Acquafredda et al., (OPERA Collaboration), JINST 4, P04018 (2009)

6. A. Aleksandrov et al., (NEWSdm Collaboration), LNGS-LOI 48/15 (2016) arXiv:1604.04199 [astro-ph.IM]

7. P. Ullio, M. Kamionkowski, JHEP 03, 049 (2001)

8. B. Morgan, A.M. Green, N.J.C. Spooner, Phys. Rev. D 71, 103507 (2005)

9. A. Alexandrov et al., Method and optical microscope for detecting particles having sub-diffractive size, $\mathrm{WO} / 2018 / 122814$ (2018)

10. M. Kimura et al., Nucl. Instr. Meth. A845, 373 (2017)

11. A. Aleksandrov et al., Astropart. Phys. 80, 16 (2016)

12. J.F. Ziegler, M. Ziegler, J. Biersack, Nucl. Instr. Meth. B268, 1818 (2010)

13. G. Cowan, K. Cranmer, E. Gross, O. Vitells, Eur. Phys. J. C 71, 1554 (2011). Erratum: Eur. Phys. J. C73, 2501 (2013)

14. L. Moneta et al., PoS ACAT2010, 057 (2010)

15. W. Verkerke, CERN-2008-001, 169 (2007)

16. R. Brun, F. Rademakers, Nucl. Instr- Meth. A389, 81 (1997)

17. A.L. Read in Workshop on confidential limits, CERN, Geneva, Switzerland 17-18 Jan 2000: Proceedings, p. 81

18. J. Billard, E. Figueroa-Feliciano, L. Strigari, Phys. Rev. D 89, 023524 (2014) 